\title{
Measurement of the Earth's Rotation Using a Chip-Based Brillouin Laser Gyroscope
}

\author{
Yu-Hung Lai ${ }^{1 \dagger}$, Myoung-Gyun Suh ${ }^{1 \dagger}$, Yu-Kun Lu${ }^{1}$, Boqiang Shen ${ }^{1}$, Qi-Fan Yang ${ }^{1}$, \\ Jiang $\mathrm{Li}^{1}$, Seung Hoon Lee ${ }^{1}$, Ki Youl Yang ${ }^{1}$, Kerry J. Vahala ${ }^{1 *}$ \\ ${ }^{1}$ T. J. Watson Laboratory of Applied Physics, California Institute of Technology, Pasadena, California 91125, \\ USA \\ *vahala@caltech.edu
}

\begin{abstract}
We report a chip-based Brillouin laser gyroscope with $0.068^{\circ} / \sqrt{\mathrm{h}}$ angular random walk and $3.6^{\circ} / \mathrm{h}$ bias stability. The device can resolve sinusoidal rotations with amplitude as low as 5\% h and is also used to measure the Earth's rotation. (C) 2019 The Author(s)

OCIS codes: (140.3945) Microcavities; (140.3370) Laser gyroscopes; (290.5900) Scattering, stimulated Brillouin.
\end{abstract}

Counter-propagating lightwaves within a closed rotating loop experience different round-trip propagation times as a result of the Sagnac effect [1]. Modern optical gyroscopes enhance this difference using long coiled optical fiber paths (fiber optic gyroscopes [2]) or by recirculation using active resonators (ring laser gyroscopes [3]). With the advent of the integrated photonics, there has been interest in creation of chip-based optical gyroscopes. Such integrated optical gyrocopes could enjoy the advantages of integration and scalable manufacturing. They would also be extremely robust and rugged for operation in environments that are challenging for existing microelectrical-mechanical (MEMs) gyroscope systems [1]. For these reasons, there has been intense interest in chipbased Sagnac gyroscopes. Compact or chip-based ring laser gyroscopes [4,5], passive resonant gyroscopes [6-9], and interferometric gyroscopes [10] have recently been reported.

Here, we demonstrate an offset counter-pumped stimulated Brillouin laser (CP-SBL) gyroscope using a 36mm diameter silica wedge disk resonator [11] (Fig. 1a). By pumping the resonator from both directions, the Brillouin phase matching condition [13] allows generation of counter-propagating SBLs within the same cavity mode (Fig. 1b). This near-degenerate mode of operation offers improved stability and low-frequency (audio-rate) readout through photo detection of the CP-SBLs. However, back-scatter induced locking creates a rotation dead band similar to that observed in commercial (non-chip) ring laser systems. Here, we show that nonzero pump detuning in the range of $1 \mathrm{MHz}\left(\Delta v_{p}\right)$ introduces dispersion from the Brillouin gain that pulls the CP-SBL modes apart to unlock the gyro readout $\left(\Delta v_{s}\right)$ (Fig. 1c). The gyro readout beat frequency has a sub-Hertz linewidth because of

a

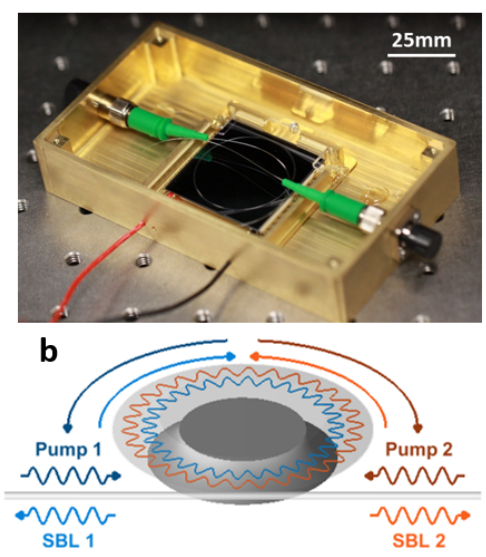

c Brillouin Lasers

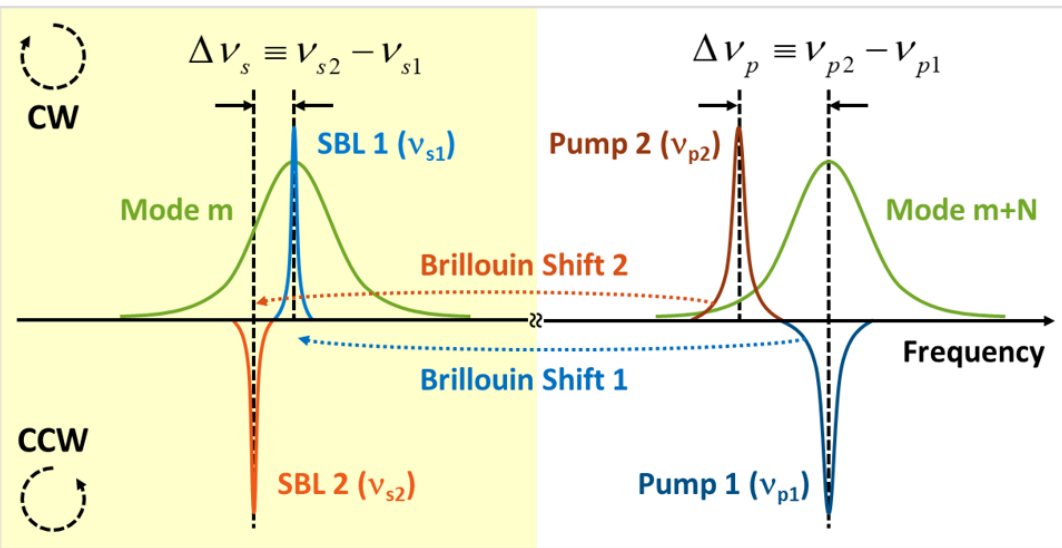

Fig. 1. Principle of the counter-pumped stimulated Brillouin laser (CP-SBL) gyroscope.

a, Photograph of a packaged 36mm-diameter silica resonator coupled with a fiber connectorized pigtail. b, The circulation directions of the pumps and their corresponding SBLs. c, Spectral diagram of offset counter-pumping. The nonzero pump detuning $\left(\Delta v_{p}\right)$ unlocks the gyro readout $\left(\Delta v_{s}\right)$ through Brillouin gain induced dispersion. 

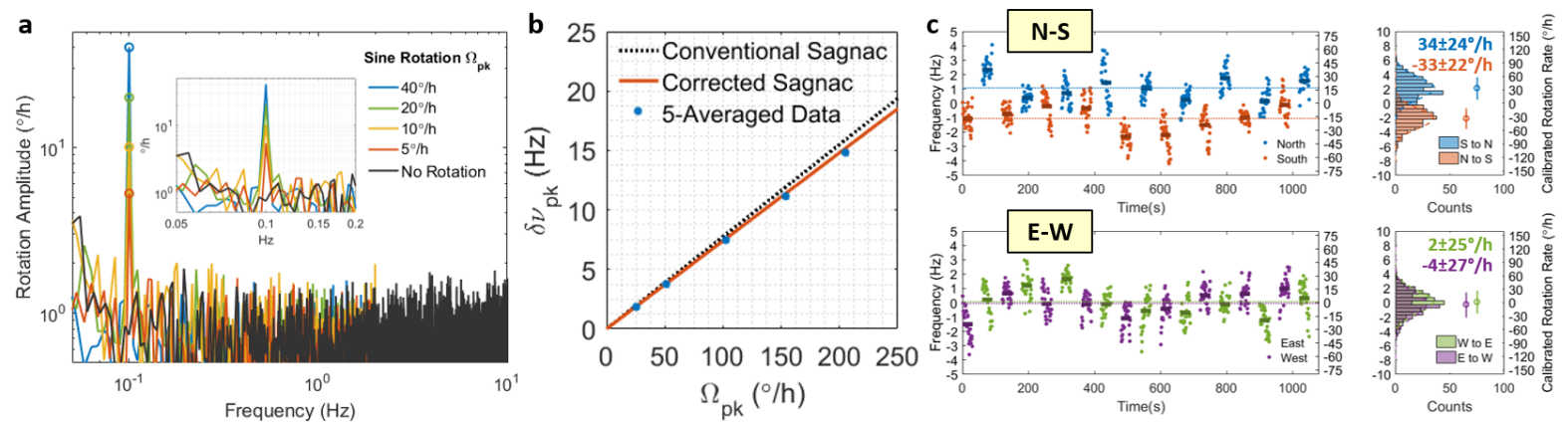

Fig. 2. Performance of the CP-SBL gyroscope. a, The FFT spectrum of the $36 \mathrm{~mm}$-gyro readout under sinusoidal rotation at $0.1 \mathrm{~Hz}$. The spectral peaks at $0.1 \mathrm{~Hz}$ resolve a series of different modulation rate amplitudes (see Legend). The minimum resolved amplitude is $5^{\circ} / \mathrm{h}$. Inset: zoom-in of the gyroscope signal. $\mathbf{b}$, Comparison of Sagnac scale factor with rotation measurements in panel a. A smaller Sagnac factor is measured due to the mode-pulling correction. c, Sagnac shift frequency versus time generated by switching the axis of the gyroscope between North and South (N-S, top) and East and West (E-W, bottom). The resulting angular-rate change for N-S is close to twice the Earth's rotation rate $\left(2 \times 15^{\circ} / \mathrm{h}\right)$ as shown in the histogram at the right. On the other hand, the E$\mathrm{W}$ measurement shows near zero change. Both measurements have similar residual long-term drift from the environment.

the high optical Q factor of the resonator [13,14]. Moreover, co-lasing of the clockwise and counter-clockwise Stokes waves suppresses common-mode drift in the beat signal [13]. The Allan deviation of the gyro shows $0.068^{\circ} / \sqrt{\mathrm{h}}$ angular random walk (ARW) and $3.6^{\circ} / \mathrm{h}$ bias stability when using a $36 \mathrm{~mm}$ diameter resonator. A smaller diameter resonator $(18 \mathrm{~mm})$ was also measured to characterize the effect of resonator area and mode volume on performance.

We also tested the performance of the CP-SBL gyroscope by applying an external sinusoidal rotation similar to the configuration in Ref. [4]. The gyroscope achieves a rotation amplitude sensitivity as low as $5^{\circ} / \mathrm{h}$ (Fig. 2a). In addition, by applying large rotation amplitudes, we confirm that the dispersion from the Brillouin lasing process introduces an additional mode-pulling correction factor to the conventional Sagnac factor (Fig. 2b). As a additional test, the Earth's rotation was measured by switching the gyroscope axis between North and South directions (Fig. 2c). As a control test, the gyroscope axis was also switched between East and West directions to verify no rotation. This is the first time that the Earth's rotation has been measured using a chip-based optical gyroscope. Our results are encouraging for the development of chip-based Brillouin laser gyroscopes in inertial guidance systems.

$\dagger$ These authors contributed equally to this work.

\section{References}

1. M. N. Armenise, C. Ciminelli, F. Dell'Olio, and V. M. N. Passaro, Advances in Gyroscope Technologies (Springer, 2010).

2. H. C. Lefèvre, The Fiber-Optic Gyroscope, 2nd Ed. (Artech House, 2014).

3. W. W. Chow, et. al., Reviews of Modern Physics 57, 61-104 (1985).

4. J. Li, M.-G. Suh, and K. J. Vahala, Optica 4, 346-348 (2017).

5. S. Gundavarapu, et. al., Nature Photonics 13, 60-67 (2019).

6. W. Liang, et. al., Optica 4, 114-117 (2017).

7. J. Zhang, H. Ma, H. Li, and Z. Jin, Opt. Lett. 42, 3658-3661 (2017).

8. P. P. Khial, A. D. White, and A. Hajimiri, Nature Photonics 12, 671-675 (2018).

9. S. Maayani, et. al., Nature 558, 569-572 (2018).

10. S. Gundavarapu, et. al., J. Light. Technol. 36, 1185-1191 (2018)

11. H. Lee, T. Chen, J. Li, K. Y. Yang, S. Jeon, O. Painter, and K. J. Vahala, Nature Photonics 6, 369 (2012).

12. M. Niklés, L. Thévenaz, and P. A. Robert, J. Lightwave Technol. 15, 1842-1851 (1997).

13. J. Li, H. Lee, T. Chen, and K. J. Vahala, Opt. Express 20, 20170-20180 (2012).

14. M.-G. Suh, Q.-F. Yang, and K. J. Vahala, Phys. Rev. Lett. 119, 143901 (2017). 